\title{
INFLUÊNCIA DO ARRANJO DOMICILIAR NAS CONDIÇÕES DE SAÚDE E NA QUALIDADE DE VIDA DOS IDOSOS RESIDENTES NA ZONA RURAL*
}

\section{INFLUENCE OF HOUSEHOLD ARRANGEMENT IN HEALTH CONDITIONS AND QUALITY OF LIFE OF ELDERLY RESIDENTS IN RURAL AREA}

\author{
INFLUENCIA DE PLAN DOMÉSTICO EN LAS CONDICIONES DE SALUD Y \\ CALIDAD DE VIDA DE LOS RESIDENTES EN EL ÁREA RURAL MAYORES
}

\author{
Camila Romanato Ribeiro ${ }^{1}$, Darlene Mara Santos Tavares ${ }^{2}$
}

\section{RESUMO}

Objetivo: Os objetivos foram descrever as características sócio demográficas e econômicas dos idosos, verificar a associação do arranjo domiciliar com as condições de saúde e qualidade de vida. Método: Estudo tipo inquérito domiciliar, analítico, transversal, observacional, realizado com 850 idosos residentes na zona rural do município de Uberaba MG, divididos em moram só e moram acompanhados. Instrumentos utilizados: MEEM, OARS, WHOQUOL- BREF, WHOQUOL-OLD. Resultados: Análises dos dados: estatística descritiva, teste qui-quadrado e t-Student $(\mathrm{p}<0,005)$ através do programa SPSS versão 17.0. Quanto à depressão não houve diferença significativa entre os grupos. $\mathrm{Na} \mathrm{QV}$ os idosos que moram só apresentaram maior escore na faceta morte $(\mathrm{p}=0,058)$ e menor na intimidade $(\mathrm{p}<0,001)$. Conclusão: Os idosos da zona rural que moram acompanhados apresentaram melhores condições de saúde e maior escore de qualidade de vida. Os resultados contribuem para a implementação de ações em saúde, de acordo com as especificidades dos idosos.

Descritores: Idoso; Depressão; Qualidade de vida; População Rural; Enfermagem geriátrica.

\begin{abstract}
Objective: The objectives were to describe the socio demographic and economic characteristics of the elderly, verifying the association of the home arrangement with the health conditions and quality of life. Method: A household, analytical, cross-sectional, observational study was carried out with 850 elderly people living in the rural area of the city of Uberaba MG, divided into households and living with them. Instruments used: MEEM, OARS, WHOQUOL-BREF, WHOQUOL-OLD. Results: Data analysis: descriptive statistics,

\footnotetext{
${ }^{1}$ Enfermeira Mestre em Atenção a Saúde pela Universidade Federal do Triângulo Mineiro (UFTM). Uberaba, Minas Gerias, Brasil. E-mail: camilaromanatoribeiro@gmail.com.br.

${ }^{2}$ Doutora em Enfermagem. Professora Associada do Departamento de Enfermagem em Educação e Saúde Comunitária. Pró-Reitora de Pesquisa e Pós-Graduação Universidade Federal do Triângulo Mineiro da Universidade Federal do Triângulo Mineiro (UFTM). Uberaba, Minas Gerais, Brasil. E-mail: darlenetavares@enfermagem.uftm.edu.br

*Pesquisa decorrente de projeto financiado pelo Conselho Nacional de Desenvolvimento Científico e Tecnológico- CNPq.
} 
chi-square test and Student's t-test $(\mathrm{p}<0.005)$ through the SPSS program version 17.0. As for depression there was no significant difference between the groups. In the QoL, the elderly who lived only presented higher scores in the death facet $(\mathrm{p}=0.058)$ and lower in the intimacy ( $p<0.001)$. Conclusion: Elderly people living in rural areas showed better health and a higher quality of life score. The results contribute to the implementation of health actions, according to the specificities of the elderly.

Descriptors: Elderly; Depression; Quality of life; Rural population; Geriatric Nursing.

\section{RESUMEN}

Objetivo: Los objetivos fueron describir las características socio demográficas y económicas de los ancianos, verificando la asociación del arreglo domiciliar con las condiciones de salud y calidad de vida. Método: Estudio tipo encuesta domiciliaria, analítica, transversal, observacional, realizada con 850 ancianos residentes en la zona rural del municipio de Uberaba MG, divididos en moras sólo y viven acompañados. Instrumentos utilizados: MEEM, OARS, WHOQUOL- BREF, WHOQUOL-OLD. Resultados: Análisis de los datos: estadística descriptiva, prueba qui-cuadrado y t-Student $(\mathrm{p}<0,005)$ a través del programa SPSS versión 17.0. En cuanto a la depresión no hubo diferencia significativa entre los grupos. En la QV los ancianos que viven sólo presentaron mayor puntuación en la faceta muerte ( $\mathrm{p}=$ $0,058)$ y menor en la intimidad ( $p<0,001)$. Conclusión: Los ancianos de la zona rural que viven acompañados presentaron mejores condiciones de salud y mayor puntuación de calidad de vida. Los resultados contribuyen a la implementación de acciones en salud, de acuerdo con las especificidades de los ancianos.

Descriptores: Edad avanzada; Depresión; Calidad de vida; Población rural; Enfermería Geriátrica.

\section{INTRODUÇÃO}

O envelhecimento da população brasileira é uma característica marcante da transição da estrutura etária do país. $^{1}$ Espera-se que, em 2020, os idosos brasileiros sejam, aproximadamente, $15 \%$ da população total. ${ }^{2}$ Dados da Organização Mundial de Saúde estimam que, no ano de 2050, existirão cerca de 2 bilhões de indivíduos com 60 anos ou mais no mundo, a maioria vivendo em países em desenvolvimento O Brasil, que ocupa a $16^{\mathrm{a}}$ posição entre os países com maior

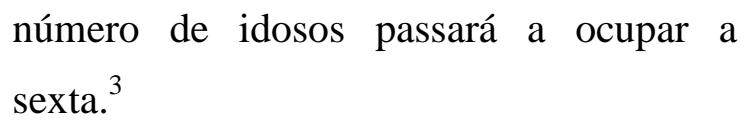
número de idosos passará a ocupar a sexta. $^{3}$

A população idosa brasileira está representada por $12,32 \%$ do total de habitantes. $\mathrm{O}$ estado de Minas Gerais possui uma população de 19.597.330 habitantes, sendo $12,2 \%$ composta por pessoas com 60 anos ou mais de idade. Já o município de Uberaba, local de realização desta pesquisa, possui $12,6 \%$ de idosos, sendo que $20 \%$ dos idosos residem na área rural. ${ }^{4}$

Os idosos que residem em áreas rurais apresentam maior dificuldade no 
acesso aos serviços de saúde devido a grandes distâncias das unidades de atendimento e carência nos meios de transporte. Desse modo, as condições de saúde dos idosos podem ser agravadas interferindo na sua qualidade de vida (QV). ${ }^{5}$

Definir qualidade de vida na velhice é um trabalho complexo, uma vez que se associa às condições biológicas, sociais e psicológicas a que o idoso foi exposto ao longo da vida. ${ }^{6}$

A influência do arranjo moradia no cuidado à saúde dos idosos é um assunto pouco estudado no mundo, especialmente com idosos residentes na zona rural. ${ }^{7}$

Dessa forma o objetivo desse estudo foi verificar a associação do arranjo domiciliar com as condições de saúde, o indicativo de depressão e a qualidade de vida de idosos residentes na zona rural, a fim de contribuir com a ampliação do conhecimento na temática e subsidiar o desenvolvimento de ações em saúde para a promoção do envelhecimento saudável.

\section{METODOLOGIA}

Essa pesquisa faz parte de um estudo maior, tipo inquérito domiciliar, analítico, transversal e observacional, denominado "Saúde e qualidade de vida da população idosa da zona rural de Uberaba”. A população foi constituída por
1.297 idosos cadastrados nas Equipes de Saúde da Família (ESF) da zona rural de Uberaba-MG, que cobrem $100 \%$ da população. A Secretaria Municipal de Saúde ofereceu as listas contendo os nomes e endereços dos idosos por ESF, em junho de 2010.

Para a organização da atenção à saúde, o espaço rural do referido município está dividido em três Distritos Sanitários (DS) atendidos por quatro equipes de Estratégia Saúde da Família (ESF), que cobrem $100 \%$ das áreas de abrangência: Borgico/Calcário (DSII); Baixa/Capelinha (DSII), Santa Rosa (DSIII) e Ponte Alta/Peirópolis (DSI).

Estabeleceram como critérios de inclusão: idosos com 60 anos ou mais de idade; residentes na zona rural do município de Uberaba-MG; sem declínio cognitivo e que aceitaram participar da pesquisa. Foram excluídos 447 idosos, sendo que 117 (9\%) tinham mudado de endereço, $105(8,1 \%)$ apresentaram declínio cognitivo, 75 (5,8\%) recusaram participar, $57 \quad(4,4 \%)$ não foram encontrados após três tentativas do entrevistador, $11(3,8 \%)$ tinham ido a óbito, três $(0,2 \%)$ encontravam-se hospitalizados e $79(6,1 \%)$ devido a outros motivos, como residir na cidade. Participaram do estudo 833 idosos, distribuídos em dois grupos: 119 idosos que moravam sozinhos e 714 idosos que 
moravam acompanhados. As entrevistas foram realizadas por 14 entrevistadores, devidamente orientados e treinados quanto à maneira de abordar o entrevistado, o preenchimento correto do instrumento e sobre as questões éticas relacionadas à pesquisa. A coleta dos dados ocorreu no período de junho de 2010 a março de 2011. Contou-se com a colaboração dos Agentes Comunitários de Saúde, os quais acompanharam os entrevistadores até o domicílio do idoso.

As entrevistas foram revisadas por supervisores de campo, que verificaram questões incompletas e inconsistência das respostas. Quando necessário, a entrevista foi devolvida ao entrevistador para que fosse realizado o preenchimento adequado.

Antes de realizar a entrevista aplicou-se a avaliação cognitiva por meio do Mini Exame de Estado Mental (MEEM), traduzido e validado no Brasil. Os escores variam de 0 a 30 pontos, sendo os pontos de corte: 13 para analfabetos, 18 para escolaridade de 1 a 11 anos e 26 para escolaridade superior a 11 anos. $^{8}$

Para a caracterização dos dados sociodemográficos, utilizou-se de instrumento estruturado baseado no questionário Olders Americans Resoucers and Services (OARS), adaptado à realidade brasileira. ${ }^{9}$ As variáveis socioeconômicas e demográficas foram: sexo; faixa etária (em anos); estado conjugal; escolaridade; renda individual (em salários mínimos); procedência de recursos financeiros; razão de aposentadoria; atividade profissional atual; satisfação das necessidades básicas em relação à situação econômica; comparação da situação econômica com outros idosos da mesma idade, moradia e arranjo domiciliar.

As variáveis para avaliação da $\mathrm{QV}$ do WHOQOL-BREF foram os quatro domínios: físico (dor e desconforto; energia e fadiga; sono e repouso; atividades da vida cotidiana; dependência de medicação ou tratamentos e capacidade de trabalho); psicológico (sentimentos positivos; pensar, aprender, memória e concentração; autoestima; imagem corporal e aparências; sentimentos negativos; espiritualidade, religiosidade e crenças pessoais); relações sociais (relações pessoais; suporte social e atividade sexual); meio ambiente (segurança física e proteção; ambiente no lar; recursos financeiros; cuidados de saúde e sociais; disponibilidade e qualidade; oportunidade de adquirir novas informações e habilidades; participação e oportunidade de recreação/lazer; ambiente físico: poluição, ruído, trânsito, clima e transporte). ${ }^{10}$

Já para o WHOQOL-OLD foram seis facetas, a saber: funcionamento dos sentidos (avalia o funcionamento sensorial e o impacto da perda das habilidades 
sensoriais na QV); autonomia (refere-se à independência na velhice, descreve até que ponto se é capaz de viver de forma autônoma e tomar suas próprias decisões); atividades passadas, presentes e futuras (descreve a satisfação sobre conquistas na vida e coisas a que se anseia); participação social (participação em atividades cotidianas, especialmente na comunidade); morte e morrer (preocupações, inquietações e temores sobre a morte e morrer), e intimidade (avalia a capacidade de se ter relações pessoais e íntimas). As respostas dos idosos sobre a QV se referem às últimas duas semanas que antecederam as entrevistas. $^{11}$

Conforme as entrevistas foram realizadas, foi conduzida à revisão e à codificação dos dados. Foi construído um banco de dados eletrônico, no programa Excel® onde os dados foram processados por dupla entrada, para verificação da existência de registros duplicados, nomes diferentes e digitação incorreta entre as duas bases de dados. Os dados inconsistentes foram verificados na entrevista original e realizou-se a sua correção.

Os dados foram transportados para o software Statistical Package for Social Science (SPSS), versão 17.0, para análise.
Para atender o primeiro objetivo, foi utilizada análise estatística descritiva com a construção de tabela de frequência simples para as variáveis categóricas.

Quanto ao segundo objetivo, foi aplicado o teste qui-quadrado para verificar associação entre o arranjo de moradia e as condições em saúde. Para a análise de qualidade de vida e o arranjo de moradia, foi utilizado o teste t-Student. Para todos os testes, adotou-se como significância quando $\mathrm{p}<0,05$.

Este projeto obteve aprovação pelo Comitê de Ética em Pesquisa da Universidade Federal do Triângulo Mineiro, protocolo $\mathrm{n}^{\circ}$ 1477. Os sujeitos foram contatados, em seus domicílios, aos quais se apresentaram os objetivos, o Termo de Consentimento Livre e Esclarecido e oferecido às informações pertinentes. Somente após a anuência do entrevistado e assinatura do referido Termo foi conduzida a entrevista.

\section{RESULTADOS}

Na Tabela 1, a seguir, encontram-se as características socioeconômicas e demográficas da população idosa estudada.

Tabela 1 -Distribuição da frequência das variáveis sociodemográficas e econômicas, Segundo o arranjo domiciliar dos idosos residentes na zona rural de Uberaba-MG, 2012. 


\begin{tabular}{|c|c|c|c|c|}
\hline $\begin{array}{c}\text { domiciliar } \\
\text { Variáveis }\end{array}$ & & $\begin{array}{c}\text { Mora só } \\
\text { n }(\%)\end{array}$ & $\begin{array}{c}\text { Mora acompanhado } \\
\text { n (\%) }\end{array}$ & $\begin{array}{l}\text { Total } \\
\text { n }(\%)\end{array}$ \\
\hline \multirow[t]{2}{*}{ Sexo } & Masculino & $67(56,3)$ & $366(51,3)$ & $433(52)$ \\
\hline & Feminino & $52(43,7)$ & $348(48,7)$ & $400(48)$ \\
\hline \multirow[t]{3}{*}{ Faixa etária } & $60-70$ & $61(51,3)$ & $422(61,9)$ & $483(60,4)$ \\
\hline & $70 \vdash-80$ & $46(38,7)$ & $212(29,7)$ & $258(31)$ \\
\hline & 80 ou mais & $12(10,1)$ & $60(8,4)$ & $72(8,6)$ \\
\hline \multirow[t]{4}{*}{ Escolaridade } & Analfabeto & $37(31,9)$ & $165(24)$ & $202(25,2)$ \\
\hline & $1-4$ & $33(28,4)$ & $218(31,7)$ & $251(31,3)$ \\
\hline & 4 -8 & $42(36,2)$ & $264(38,4)$ & $306(38,1)$ \\
\hline & 8 ou mais & $4(3,4)$ & $40(5,8)$ & $44(5,5)$ \\
\hline \multirow[t]{4}{*}{ Estado conjugal } & Solteiro & $26(21,8)$ & $35(4,9)$ & $61(7,3)$ \\
\hline & Casado & 0 & $555(77,7)$ & $555(66,6)$ \\
\hline & Viúvo & $58(48,7)$ & $103(14,4)$ & $161(19,3)$ \\
\hline & sep/desq/div & $35(29,4)$ & $21(2,9)$ & $56(6)$ \\
\hline \multirow[t]{6}{*}{ Renda individual } & sem renda & $6(5)$ & $80(11,2)$ & $86(10,3)$ \\
\hline & $<1$ & $5(4,2)$ & $25(3,5)$ & $30(3,6)$ \\
\hline & $1-3$ & $72(60,5)$ & $334(46,8)$ & $406(48,8)$ \\
\hline & $3+5$ & $30(25,2)$ & $219(30,7)$ & $249(29,9)$ \\
\hline & 5 ou mais & $5(4,2)$ & $38(5,3)$ & $43(5,2)$ \\
\hline & não sabe & $1(0,8)$ & $17(2,4)$ & $18(2,2)$ \\
\hline \multirow[t]{9}{*}{ Recurso financeiro } & Aposentadoria/outro & $82(68,9)$ & $498(70)$ & $580(69,8)$ \\
\hline & Pensão & $17(14,3)$ & $58(8,1)$ & $75(9,0)$ \\
\hline & renda/aluguel e outro & $1(0,8)$ & $13(1,9)$ & $14(1,7)$ \\
\hline & Doação (família) & 0 & $3(0,4)$ & $3(0,3)$ \\
\hline & Trabalho contínuo e outro & $10(8,4)$ & $52(7,3)$ & $62(7,4)$ \\
\hline & Trabalho eventual & $4(3,4)$ & $10(1,4)$ & $14(1,7)$ \\
\hline & Renda mensal vitalícia & 0 & $1(0,1)$ & $1(0,1)$ \\
\hline & Aplicação financeira & 0 & 0 & 0 \\
\hline & Sem rendimento próprio & $3(2,5)$ & $72(10,1)$ & $75(12,6)$ \\
\hline
\end{tabular}

Fonte: a autora, 2011.

Em relação ao sexo, predominou o masculino (52\%), com percentual superior 
para os que moram só $(56,3 \%)$ em relação aos que vivem acompanhados (51,3\%). No que concerne à faixa etária, a maioria encontrava-se entre 60-70 anos (60,4\%). Contudo, o percentual de idosos que moravam só $(51,3 \%)$ era menor do que aqueles que residiam acompanhados $(61,9 \%)$ (Tabela 1).

Destaca-se, neste estudo, o maior percentual de idosos mais velhos morando só, representados por $38,7 \%$ para os com 70-80 anos e 10,1\% para os de 80 anos e mais. Estes achados são satisfatórios, considerando-se que, no Brasil, morar só após os 60 anos pode ser entendido como consequência de um envelhecimento bem sucedido, acompanhado de boas condições de saúde, integração social, desmistificando, assim, a ideia de abandono e solidão.

Referente à escolaridade, ambos os grupos apresentaram, em sua maioria, 4-8 anos de estudo, representando 36,2\% para os idosos que moravam só e $38,4 \%$ para os que moravam acompanhados.

Quanto ao estado conjugal, o maior percentual de idosos que moravam só eram viúvos $(48,7 \%)$, enquanto os que moravam acompanhados eram casados $(77,7 \%)$ (Tabela 1). No que se refere à renda individual, a maioria recebia 1-3 salários mínimos mensais $(48,8 \%)$, entretanto, o percentual era superior para os idosos que moravam só $(60,5 \%)$ do que para os acompanhados (46,8\%) (Tabela 1).

No que se refere à renda individual, a maioria recebia 1-3 salários mínimos mensais $(48,8 \%)$, entretanto, o percentual era superior para os idosos que moravam só $(60,5 \%)$ do que para os acompanhados $(46,8 \%)$. A procedência dos recursos financeiros, em sua maioria, era da aposentadoria $(69,8 \%)$, distribuídos em $70 \%$ para os idosos que moravam acompanhados e $68,9 \%$ para os que residiam só (Tabela 1).

A atividade profissional predominante foi a de dona de casa, significando $35,3 \%$ para os idosos que moravam só e $41,1 \%$ para aqueles que moravam acompanhados. Destacou-se que $31,9 \%$ dos idosos que moram só são trabalhadores rurais.

A maioria dos idosos eram aposentados, em ambos os grupos. A razão da aposentadoria tanto os idosos que moravam só $(44,4 \%)$ como para os que moravam acompanhados (39,3\%), apresentou maior percentual para a idade.

Os idosos que moram só $(56,6 \%)$ e acompanhados $(53,5 \%)$ consideraram sua situação econômica é igual à de outras pessoas de mesma idade. Tanto os idosos que moram só $(58,8 \%)$ quanto os que moram acompanhados $(71,1 \%)$ possuem casa própria (Tabela 1). Destaca-se que $31,9 \%$ dos idosos que moravam sozinhos residiam em casa cedida. 
$\mathrm{Na}$ Tabela 2 apresenta-se a associação das condições de saúde segundo o arranjo domiciliar dos idosos residentes na área rural.

Tabela 2- Fatores associados às condições de saúde segundo arranjo domiciliar de Idosos residentes na zona rural do município de Uberaba-MG, 2012.

\begin{tabular}{|c|c|c|c|c|c|}
\hline Condições de Saúde & $\begin{array}{c}\text { Mora só } \\
\mathrm{n}(\%)\end{array}$ & $\begin{array}{c}\text { Mora acompanhado } \\
\mathrm{n}(\%)\end{array}$ & $\begin{array}{l}\text { Total } \\
\mathrm{n}(\%)\end{array}$ & $\chi^{2}$ & $p$ \\
\hline Auto percepção da saúde & & & & 1,37 & 0,849 \\
\hline Péssima & $3(2,5)$ & $20(2,8)$ & $23(2,8)$ & & \\
\hline Má & $5(4,2)$ & $27(3,8)$ & $32(3,8)$ & & \\
\hline Regular & $50(42)$ & $323(45,2)$ & $373(44,8)$ & & \\
\hline Boa & $51(42,9)$ & $270(37,8)$ & $321(38,5)$ & & \\
\hline Ótima & $10(8,4)$ & $74(10,4)$ & $84(10,1)$ & & \\
\hline Auto percepção da visão & & & & 13,226 & 0,010 \\
\hline Cega & 0 & 0 & 0 & & \\
\hline Péssima & $8(6,8)$ & $29(4,1)$ & $107(12,9)$ & & \\
\hline Ruim & $13(11)$ & $94(13,2)$ & $358(43)$ & & \\
\hline Regular & $37(31,4)$ & $321(45)$ & $297(35,7)$ & & \\
\hline Boa & $57(48,3)$ & $240(33,6)$ & $33(4)$ & & \\
\hline Excelente & $3(2,5)$ & $30(4,2)$ & $37(4,4)$ & & \\
\hline Auto percepção da audição & & & & 0,874 & 0,832 \\
\hline Surdo & 0 & $2(3)$ & $2(2)$ & & \\
\hline Ruim & $24(20,3)$ & $130(18,3)$ & $154(18,6)$ & & \\
\hline Boa & $79(66,9)$ & $487(68,6)$ & $566(68,4)$ & & \\
\hline Excelente & $15(12,7)$ & $91(12,8)$ & $106(12,8)$ & & \\
\hline Uso regular de medicação & & & & 3,159 & 0,206 \\
\hline Sim receitado pelo médico & $84(70,6)$ & $516(72,3)$ & $600(72)$ & & \\
\hline Sim por conta própria & $8(6,7)$ & $24(3,4)$ & $32(3,8)$ & & \\
\hline Não & $27(22,7)$ & $174(24,4)$ & $201(24,1)$ & & \\
\hline Prática de atividade física & & & & 3,491 & 0,062 \\
\hline Sim & $39(34,2)$ & $184(25,8)$ & $223(27)$ & & \\
\hline Não & $75(65,8)$ & $528(74,2)$ & $603(73)$ & & \\
\hline Tabagismo & & & & 8,902 & 0,003 \\
\hline Sim & $33(28,4)$ & $120(16,9)$ & $153(18,5)$ & & \\
\hline Não & $83(71,6)$ & $592(83,1)$ & $675(81,5)$ & & \\
\hline Ingesta de bebida alcoólica & & & & 4,757 & 0,446 \\
\hline Diariamente & $1(0,8)$ & $35(4,9)$ & $36(4,3)$ & & \\
\hline 1 a 3 vezes por semana & $10(8,5)$ & $71(9,9)$ & $81(9,7)$ & & \\
\hline 4 a 6 vezes por semana & $2(1,7)$ & $8(1,1)$ & $10(1,2)$ & & \\
\hline 1 a 3 vezes por mês & $12(10,2)$ & $66(9,2)$ & $78(9,4)$ & & \\
\hline Menos de 1 vez por mês & $8(6,8)$ & $41(5,7)$ & $49(12,5)$ & & \\
\hline
\end{tabular}

Fonte: autora, 2011.

A maioria dos idosos que moravam só consideraram sua saúde boa $(42,9 \%)$ e regular $(42 \%)$, enquanto os que moravam acompanhados avaliam-na como regular $(45,2 \%)$ e boa $(37,8 \%)$, sem diferença estatística significativa entre os grupos $(\chi 2=1,37 ; p=0,849)$. 
Verificou-se

diferença

significativa na auto-percepção da visão entre os grupos, sendo que os idosos que moravam só consideravam-na boa e os que moravam acompanhados, regular $(\chi 2=13,226 ; p=0,010)$ (Tabela 2).

A audição foi percebida pelos idosos que moravam só $(66,9 \%)$ e acompanhados $(68,6 \%)$ como boa, sem diferença significativa entre os grupos ( $\chi 2=0,874 ; p=0,832)$ (Tabela 2).

A maioria dos idosos fazia uso regular de remédios receitados pelo médico, com percentuais menores para os que moravam só $(70,6 \%)$ em relação aos que moravam acompanhados (72,3\%), sem diferença estatística significativa entre os grupos $(\chi 2=3,159 ; p=0,206)$.

Os idosos que moram sozinhos apresentaram maior percentual na prática de atividade física $(34,2 \%)$ em relação aos que moravam acompanhados (25,8\%), porém, não se obteve diferença significativa entre os grupos $(\chi 2=3,491$; $p=0,06)$ (Tabela 2).

Houve maior proporção de idosos que moravam só que eram tabagistas comparados aos que moravam acompanhados $(\chi 2=8,902 ; p=0,003)$. Em ambos os grupos a maioria relatou não fazer uso de bebida alcoólica; no entanto, percentual inferior foi encontrado entre os idosos que moravam só $(67,4 \%)$ em relação aos acompanhados (69,0\%), sem diferença estatística significativa $(\chi 2=4,757 ; p=0,440)$.

Em relação à consulta médica mensal, ambos os grupos relataram não comparecer, representando $68,1 \%$ para os idosos que moravam só e $75,3 \%$ para os acompanhados, sem diferença estatística entre os grupos $(\chi 2=2,718 ; p=0,09)$.

Em relação à depressão, 24,4\% dos idosos que moravam só e 21,4\% dos idosos que moravam acompanhados apresentaram o indicativo para a doença. Não foi verificada diferença significativa entre os grupos $\left(\chi^{2}=0,517 ; p=0,472\right)$. Verificou-se maior proporção de idosos que moravam só auto-referindo catarata em relação aos idosos que moravam acompanhados $\left(\chi^{2}=\right.$ $3,734 ; p=0,053)$.

$\mathrm{Na}$ Tabela 3, a seguir, encontram-se os escores de QV mensurado pelo WHOQOL-BREF.

Tabela 3 - Qualidade de vida dos idosos, residentes na zona rural, avaliado pelo WHOQOLBREF, segundo arranjo domiciliar, Uberaba MG, 2012.

\begin{tabular}{lll}
\hline Qualidade de vida & Mora Só & Mora Acompanhado
\end{tabular}




\begin{tabular}{lcccccc}
\hline & Media & DP & Media & DP & $t$ & $P$ \\
\hline WHOQOOL- BREF & & & & & & \\
\hline Físico & 69,2 & 15,56 & 69,05 & 15,41 & 0,376 & 0,707 \\
\hline Psicológico & 70,23 & 13,66 & 70,05 & 13,11 & 0,139 & 0,889 \\
\hline Relações Sociais & 72,26 & 14,23 & 74,10 & 12,29 & 1,470 & 0,142 \\
\hline Meio ambiente & 63,23 & 12,90 & 63,36 & 11,4 & 0,110 & 0,912 \\
\hline
\end{tabular}

Não se observou diferença significativa entre os grupos em nenhum domínio de QV mensurado pelo WHOQOOL-BREF, Tabela 4. Em ambos os grupos, o maior escore de QV foi no domínio relações sociais.

$\mathrm{Na}$ Tabela 4, a seguir, encontram-se os escores de QV, WHOQOL-OLD, da população estudada.

Tabela 4 - Qualidade de vida dos idosos, residentes na zona rural, avaliado pelo WHOQOL-OLD, segundo arranjo domiciliar, Uberaba MG, 2012.

\begin{tabular}{lcccccc}
\hline Qualidade de vida & Mora Só & \multicolumn{7}{c}{ Mora Acompanhado } \\
\hline & Media & DP & Media & DP & $t$ & $P$ \\
\hline WHOQOOL- BREF & & & & & & \\
\hline Físico & 69,2 & 15,56 & 69,05 & 15,41 & 0,376 & 0,707 \\
\hline Psicológico & 70,23 & 13,66 & 70,05 & 13,11 & 0,139 & 0,889 \\
\hline Relações Sociais & 72,26 & 14,23 & 74,10 & 12,29 & 1,470 & 0,142 \\
\hline Meio ambiente & 63,23 & 12,90 & 63,36 & 11,4 & 0,110 & 0,912 \\
\hline
\end{tabular}

Houve maior proporção de idosos que moram só com maior escore de QV na faceta morte e morrer em comparação aos que moram acompanhados $(p=0,058)$. Os idosos que moravam só apresentaram menor escore de QV na faceta intimidade em relação aos que moravam acompanhados $(p<0,001)$.

Os idosos que moravam só apresentaram menor escore de QV na faceta intimidade em relação aos que moravam acompanhados $\quad(p<0,001)$ (Tabela 4).

\section{DISCUSSÃO}

O predomínio de idosos do sexo masculino que residem na área rural foi observado nesse estudo, corroborando com estudo desenvolvido na zona rural no interior e Minas Gerais onde 52,8\% dos idosos eram do sexo masculino ${ }^{12}$, reflexo do marco da urbanização ocorrida no Brasil na década de 70, em que se tem uma intensificação do processo de migração rural/urbano feminino, relacionado diretamente com a busca por oferta de trabalho, tanto em empresas quanto em residências, pelas mulheres.

Em relação a faixa etária, estudo desenvolvido com idosos residentes em espaço rural do interior do Rio Grande do 
Sul, corroboram com esse estudo, pois obtiveram percentual semelhante $(61,1 \%)$ embora não tenha especificação do arranjo domiciliar. ${ }^{13}$

Referente à escolaridade, ambos os grupos apresentaram, em sua maioria, 4 -8 anos de estudo, representando 36,2\% para os idosos que moravam só e $38,4 \%$ para os que moravam acompanhados (Tabela 1). Pesquisa conduzida com idosos rurais de Nova Bassano - RS, sem especificar o arranjo domiciliar, obteve porcentagem bem superior $(75 \%)$ a presente investigação. ${ }^{5}$ Os profissionais de saúde devem procurar conhecer o nível de escolaridade e possíveis dificuldades enfrentadas pelos idosos diante dos serviços de saúde. ${ }^{14}$

No que se refere à renda individual, o presente estudo observou que apesar da maioria receber de 1-3 salários mínimos mensais, o percentual era superior para os idosos que moravam só. Estudo realizado em área rural de Florianópolis, apresentou resultado semelhante ao deste estudo, fato atribuído aos benefícios pago pelo governo, denominado aposentadoria rural. $^{15}$

Ressalta-se que, durante a coleta de dados para o presente estudo observou-se que os idosos exerciam atividade com fins lucrativos como: fabricação de queijos, doces, venda de excedentes agrícolas e pecuários. Essas atividades tinham o intuito de aumentar seus rendimentos, uma vez que a maioria recebia o benefício da aposentadoria.

Quanto ao estado conjugal resultado semelhante ao nosso estudo foi encontrado para os idosos que moravam sem companheiros $(41,2 \%)$, em pesquisa realizada na zona rural do Sul do Brasil. Já para aqueles que moravam com companheiro os percentuais foram inferiores $(58,8 \%){ }^{16}$

No presente estudo a maioria dos idosos eram aposentados, em ambos os grupos. Estudo realizado com idosos da zona rural de Pelotas, apresentou resultado parcialmente semelhante ao deste estudo onde $91,8 \%$ dos idosos eram aposentados e $35,5 \%$ ainda exerciam alguma atividade laboral. $^{15}$

Observou-se também nesse estudo que tanto os idosos que moram só como os que moram acompanhados consideraram sua situação econômica igual à de outras pessoas de mesma idade. A menor idade para aposentadoria rural e a economia familiar contribuem para diferença no que se refere ao recebimento de alguns benefícios, favorecendo aqueles que residem em áreas rurais. Acredita-se que esse benéfico tenha contribuído de forma positiva para a melhoria das condições de vida desses idosos. ${ }^{4}$

Nesse estudo, houve maior proporção de idosos que moram só com 
maior escore de QV na faceta morte e morrer em comparação aos que moram acompanhados. Pesquisa realizada no interior de Minas Gerais com idosos residentes em área rural demonstrou menor escore de QV nesta faceta entre aqueles que não tinham companheiros, apresentando associação significativa $(p=0,013) .{ }^{17} \mathrm{O}$ modo como o ser humano elabora o luto e se prepara para a própria morte é particular a cada indivíduo. O enfrentamento diante da morte e do morrer se dará de acordo com as experiências vividas ao longo da vida e sua inclusão no meio social.

\section{CONSIDERAÇÕES FINAIS}

Os resultados permitiram o melhor conhecimento das características sociodemográficas, econômicas e as condições de saúde dos idosos residentes na zona rural, segundo arranjo domiciliar. Desta forma, estes resultados contribuem para o planejamento e a implementação de ações em saúde, de acordo com as especificidades dos idosos residentes na zona rural a fim de minimizar as dificuldades encontradas nessa área em relação ao acesso de serviço de saúde com a finalidade de promover a saúde e de prevenir agravos que podem interferir na QV desses idosos.

Este estudo teve como limitações ser do tipo transversal, o que não permite estabelecer causa e efeito nos resultados e ter as morbidades autorreferidas podendo estar às mesmas subdiagnosticadas.

\section{REFERÊNCIAS}

1- Pereira MG. Epidemiologia: teoria e prática. Rio Janeiro: Guanabara Koogan; 2015.

2- Ministério da Saúde (Brasil). Envelhecimento e saúde da pessoa idosa. Brasília, DF: Ministério da Saúde; 2007. (Série A. Normas e Manuais Técnicos. Cadernos de Atenção Básica, n. 19).

3- World Health Organization. Envelhecimento ativo: uma política de saúde [Internet]. Brasília: Organização Pan-Americana da Saúde; 2005 [citado em 8 mar 2012]. Disponível em: www.prosaude.org/publicacoes/diversos/en velhecimento_ativo.pdf.

4- Instituto Brasileiro de Geografia e Estatística. Síntese de indicadores sociais: uma análise das condições de vida da população brasileira. Rio de Janeiro: IBGE; 2010.

5- Bertuzzi D, Paskulin LGM, Morais EP. Arranjos e rede de apoio familiar de idosos que vivem em uma área rural. Texto \& Contexto Enferm. [Internet]. 2012 [citado em 8 mar 2012]; 21(1):158-66. Disponível em:

http://www.scielo.br/pdf/tce/v21n1/a18v21 n1.pdf

6- Chaimowicz E, Trentini CM, Fleck MPA. Qualidade de vida em idosos: conceito e investigação. In: Neri AL, organizador. Qualidade de vida na velhice: enfoque multidisciplinar. Campinas: Alínea; 2007.

7- Morais EP, Rodrigues RAP, Gerhardt TE. Os idosos mais velhos no meio rural: realidade de vida e saúde de uma população do interior gaúcho. Texto \& Contexto Enferm. 2008; 17(2):374-83.

8- Bertolucci PHF, et al. O mini exame do estado mental em uma população geral: 
impacto da escolaridade. Arq

Neuropsiquiatr. 1994; 52(1):1-7.

9- Ramos LR. Growing old in São Paulo, Brazil: assessment of health status and family support of the elderly of different socio-economic strata living in the community. [Tese]. Londres: London School of Hygiene and Tropical Medicine; 1987.

10- Fleck MPA, Louzada S, Xavier M. Aplicação da versão em português do instrumento abreviado de avaliação da qualidade de vida WHOQOL-bref. Rev Saúde Pública. 2000; 34(2):178-83.

11- Fleck MPA, Chachamovich E, Treneti C. Development and validation of the Portuguese version of the WHOQOL-OLD module. Rev Saúde Pública. 2006; 40(5): 785-91.

12- Confortin SC, Antes DL, Pessini J, Schneider IJC, d'Orsi E, Barbosa AR. Comparação do perfil socioeconômico e condições de saúde de idosos residentes em áreas predominantemente rural e urbana da Grande Florianópolis, Sul do Brasil. Cad Saúde Colet. [Internet]. 2016 [citado em 04 jun 2017]; 24(3):33038. Disponível em http://www.scielo.br/scielo.php?script=sci _arttext\&pid=S1414462X2016000300330\&lng=en

13- Rodrigues LR. Silva ATM, Dias FA, Ferreira PCS, Silva, LMA, Viana DA, et al. Perfil sociodemográfico, econômico e de saúde de idosos rurais segundo o indicativo de depressão. Rev Eletrônica Enferm. [Internet]. 2014 [citado em 04 jun 2017]; 16(2):278-85. Disponível em: https://revistas.ufg.br/fen/article/view/2078 2/16963

14- Dal Pizzol TS, Pons ES, Hugo FN, Bozzetti MC, Sousa MLR, Hilgeri JB. Uso de medicamentos entre idosos residentes em áreas urbanas e rurais de município no Sul do Brasil: um estudo de base populacional. Cad Saúde Pública. 2012; 28(1):104-14.

15- Pinto AH, Lange C, Pastore CA, Llano PMP, Castro DP, Santos F. Capacidade funcional para atividades da vida diária de idosos da Estratégia de Saúde da Família da zona rural. Ciênc Saúde Colet.

[Internet]. 2016 [citado em 29 maio 2017]; 21(11):3545-55. Disponível em: http://www.scielo.br/scielo.php?script=sci arttext\&pid=S141381232016001103545\&l $\mathrm{ng}=\mathrm{en}$

16- Tavares DMS, Gomes NC, Dias FA, Santos NMF. Fatores associados à qualidade de vida de idosos com osteoporose residentes na zona rural. Esc. Anna Nery Rev Enferm. 2012; 16(2):3718.

17- Tavares DMS, Santos LL, Dias FA, Ferreira PCS, Oliveira EA. Qualidade de vida de idosos rurais e fatores associados. Rev Enferm UFPE. 2015; 9(11):9679-87.

RECEBIDO: 26/09/2016

APROVADO: $16 / 04 / 2018$

PUBLICADO: 07/2018 